\title{
Le rôle de l'expérience de l'entrepreneur dans le niveau d'internationalisation des jeunes entreprises
}

\author{
par Mathieu Cabrol ${ }^{24}$ et Frédéric Nlemvo ${ }^{25}$
}

\begin{abstract}
Résumé
L'expérience anté-création de l'entrepreneur est de plus en plus évoquée comme l'une des raisons de l'internationalisation précoce des jeunes entreprises. En effet, de par l'expérience accumulée avant la création, l'entrepreneur développe des compétences uniques qui peuvent lui permettre de développer plus rapidement des activités sur les marchés étrangers. Dans cet article, nous analysons l'incidence de l'expérience antérieure de l'entrepreneur sur le niveau d'internationalisation des entreprises nouvelles et internationales. Les résultats de l'analyse d'un échantillon de 68 entreprises de la région Rhône-Alpes mettent en évidence l'hétérogénéité du niveau d'internationalisation de telles entreprises. Ils montrent aussi que l'expérience dans les affaires internationales permet de discriminer les entreprises qui dépendent majoritairement de l'international pour le développement de leur activité.
\end{abstract}

Abstract

The pre-founding experience of the entrepreneur is increasingly cited as a reason for the early internationalization of young firms. Thanks to the accumulated experience before founding, the entrepreneur develops unique skills that enable him to grow on international markets. In this paper, we analyze the impact of previous experience of the entrepreneur on the level of internationalization of international new ventures. The results of the analysis of a sample of 68 companies from the Rhône-Alpes region highlight the heterogeneity of the degree of internationalization of these companies. It also shows that the international business experience of the company distinguishes the companies that depend mostly on international development of their business.

L'étude des Entreprises Nouvellement Internationales (ENI) occupe une place de plus en plus prépondérante dans la littérature en entrepreneuriat international (McDougall et al., 2003). Traditionnellement, les entreprises s'internationalisent selon un développement lent et graduel (Johanson et Vahlne, 1990). Cependant, un phénomène accentué dans les années 90 montre que d'autres entreprises plus dynamiques et nouvellement créées deviennent internationales dès leur

24. MAthieu CABrol Professeur assistant, Groupe Sup de Co Montpellier, m.cabrol@supco-montpellier.fr

25. FRÉDÉRIC NLEMvo, Professeur, Reims Management School, Laboratoire ICE@RMS, frederic.nlemvo@reims-ms.fr 
création ou peu de temps après. Qualifiées de 'born global' (McKinsey et Co, 1993) ou de 'global start-ups' ou encore 'd'international new ventures' (Oviatt et McDougall, 1994), ce sont des entreprises qui, dès leur création ou peu de temps après, cherchent à tirer un avantage concurrentiel de l'utilisation de ressources et de la vente de produits dans de multiples pays (Oviatt et McDougall, 1994). Dans leur revue de la littérature portant sur la décennie 1993-2003, Rialp et al. (2005) mettent en évidence une dizaine de facteurs à l'origine de ce phénomène d'internationalisation rapide, à savoir : une vision globale de l'entrepreneur et de son équipe, l'expérience du dirigeant, un engagement managérial, l'appartenance à un réseau, une connaissance pointue du marché, la présence de ressources intangibles, une différenciation produit, une stratégie de niche, une orientation client prononcée et la flexibilité. Si nombre d'étude ${ }^{26}$ ont examiné empiriquement l'incidence de la plupart de ces facteurs sur l'émergence de telles entreprises, relativement peu ont porté spécifiquement sur l'influence de l'expérience de l'entrepreneur. Pour Autio (2005), l'expérience est au cœur du débat entre l'approche du processus d'internationalisation (Johanson et Vahlne, 1990) et de l'approche des entreprises nouvelles et internationales (Oviatt et McDougall, 1994). En effet, les connaissances expérientielles acquises sur les marchés étrangers au niveau organisationnel sont à l'origine du processus d'apprentissage à l'international (Johanson et Vahlne, 1990). L'expérience acquise au niveau individuel par l'entrepreneur et son équipe permet de s'internationaliser dès la création et de tirer un avantage concurrentiel de la mobilisation de ressources sur les marchés étrangers (Oviatt et McDougall, 1994).

Dans cet article, nous nous focalisons particulièrement sur l'expérience antécréation de l'entrepreneur, en examinant la question de recherche suivante : "L'expérience anté-création de l'entrepreneur explique-t-elle le niveau d'internationalisation d'une jeune entreprise ? ». En particulier, nous analyserons les différences existantes en termes d'expérience anté-création entre les jeunes entreprises qui orientent de façon majoritaire leurs activités à l'international (plus de $50 \%$ de chiffre d'affaires à l'international) et celles qui dépendent majoritairement de leur activité domestique (moins $50 \%$ de chiffre d'affaires réalisé à l'international). Selon la littérature en entrepreneuriat international (Rialp et al., 2005), l'orientation internationale de ces entreprises peut être attribuée aux processus cognitifs et/ou aux connaissances liées àl'expérience accumulée à partir d'activités précédentes, que ce soit les activités liées aux affaires internationales, au management ou encore à l'exercice d'une activité entrepreneuriale. Fort de cette expérience, l'entrepreneur est alors plus enclin à percevoir et à exploiter les opportunités se manifestant sur les marchés étrangers.

L'article est structuré comme suit. Dans la première partie nous étudions la perspective des entreprises nouvelles et internationales (Oviatt et McDougall,

26. Voir la synthèse de Rialp et al. (2005) 
1994), en mettant l'accent sur le rôle de l'expérience et des connaissances de l'entrepreneur dans le processus d'internationalisation des jeunes entreprises. Dans la deuxième partie, nous exposons la méthodologie adoptée pour répondre à notre question de recherche. Enfin, dans la troisième partie, nous présentons et discutons les résultats, avant de conclure en mettant aussi en évidence les limites ainsi que les nouvelles pistes de recherche susceptibles de prolonger cette recherche.

\section{Revue de la littérature}

L'internationalisation précoce des jeunes entreprises a remis en question les approches théoriques traditionnelles de l'internationalisation (McDougall et al., 1994). Aussi nous centrons notre analyse sur la perspective des entreprises nouvelles et internationales telle qu'elle a été développée initialement par Oviatt et McDougall (1994).

\subsection{Le cadre théorique des entreprises nouvelles et internationales : l'intégration de l'expérience et des connaissances de l'entrepreneur}

Dans les années 90, de nombreuses études, essentiellement empiriques, ont examiné l'internationalisation précoce d'entreprises nouvellement créées. Ces recherches suggèrent que le phénomène des ENI est de grande ampleur et qu'il se produit dans différents pays et continents. Par ailleurs, même si la majorité de ces entreprises sont actives dans les hautes technologies, d'autres secteurs plus traditionnels de l'économie y sont également représentés (Moen, 2002 ; Servais et Rasmussen, 2000).

Les fondements du cadre théorique proposé par Oviatt et McDougall $(1994,2005)$ pour expliquer ce phénomène s'appuient sur les analyses traditionnellement utilisées pour expliquer l'existence des firmes multinationales, à savoir celles des coûts de transaction, des imperfections des marchés et de l'internalisation des transactions essentielles à l'international. Ces fondements incorporent également certains concepts issus de l'entrepreneuriat, notamment la notion de contrôle de certaines ressources sans les posséder. Ils s'appuient aussi sur le champ du management stratégique en intégrant la façon dont un avantage concurrentiel est développé et soutenu, notamment à l'international. Le cadre explicatif de l'existence des ENI s'appuie ainsi sur quatre éléments distinctifs : (1) la formation organisationnelle à travers l'internalisation de certaines transactions ; (2) l'appui marqué sur des structures de gouvernance hybrides ; (3) l'établissement de l'avantage de localisation à l'étranger ; (4) le contrôle de ressources uniques (dont la connaissance). Si pour Oviatt et McDougall (2005), ces éléments restent valables pour la théorie des ENI, certaines recherches (Shrader et al., 
2000) montrent qu'un certain nombre d'entreprises utilisent moins les formes de gouvernance hybrides, comme les licences et les réseaux d'alliances, que ne le prédit le modèle. Le cadre théorique d'Oviatt et McDougall (1994) trouve ses racines dans l'approche par les ressources. Les ENI doivent disposer de ressources uniques pour générer un avantage concurrentiel soutenable à l'international. En particulier, la connaissance technologique unique dont de telles entreprises peuvent disposer les amène à concevoir une offre globale au niveau mondial de manière précoce (Autio, 2005). Knight et Cavusgil (2004), quant à eux, mettent en avant l'importance de la culture d'innovation, la connaissance et les capacités de l'entreprise pour rendre compte de son internationalisation précoce.

Contrairement à l'approche du processus d'internationalisation (Johanson et Vahlne, 1990), McDougall et al. (1994) mettent davantage en avant la personne de l'entrepreneur pour expliquer la dynamique des entreprises nouvelles et internationales. Bien souvent, l'entrepreneur démarre l'internationalisation avant même la création de l'entreprise et commence à tisser des liens à l'international pendant la gestation de l'entreprise (Cabrol etal., 2009). Ainsi doncl'idée d'effectuer des ventes à l'international fait partie du projet de création d'entreprise (Madsen et Servais, 1997). Les créateurs des ENI ainsi que leurs équipes développent des compétences internationales dès le début, en orientant l'entreprise à l'international à tous les niveaux. II en résulte comme avantage l'instauration précoce d'un esprit international au tout début du développement de l'entreprise (McDougall et al., 1994). Ainsi les créateurs de ces entreprises se prédisposent à acquérir les compétences dans le domaine des affaires internationales et cherchent à éviter le phénomène de 'path-dependency' des compétences domestiques, dont l'entreprise peut ne pas sortir du fait de l'existence de forces d'inertie. Ainsi donc, la décision de s'internationaliser est décrite comme étant entrepreneuriale et proactive ; les entreprises démarrent l'internationalisation pour la poursuite d'opportunités de croissance (Sapienza et al., 2005).

Oviatt et McDougall $(1994,1995)$ mettent en évidence le besoin de redéfinir les types de compétences et de connaissances organisationnelles requises pour gérer l'internationalisation, dans la mesure où un effet levier de l'expérience dans l'internationalisation estsusceptible d'exister. Ces auteurs considèrentle lancement d'opérations transnationales comme une compétence organisationnelle qui peut être apprise. De ce fait, elle peut constituer une partie de la dotation initiale en ressources de l'entreprise, notamment à travers l'expérience de l'entrepreneur. Cette distinction a été établie subséquemment entre la 'connaissance du marché étranger' et la 'connaissance de l'internationalisation' ou la 'connaissance sur la manière de s'internationaliser' (Eriksson et al., 2000). La perspective des ENI met l'accent sur le transfert, la nature et les types d'expériences d'internationalisation ainsi que de compétences nécessaires pour rendre compte de l'internationalisation des entreprises récemment créées. 


\subsection{Rôle de l'expérience de l'entrepreneur, de ses compétences et de ses ressources dans le processus d'internationalisation}

Les recherches en entrepreneuriat international reconnaissent unanimement (Rialp et al., 2005) que les ENI possèdent des ressources et compétences inégalables qui leur servent de tremplin pour l'international (Weerawardena et al., 2007). En effet, l'approche par les ressources (Barney, 1991) permet de mieux comprendre la formation de l'avantage concurrentiel et le démarrage précoce de ces entreprises à l'international. L'accumulation des connaissances autour de la personne du dirigeant ou de l'équipe dirigeante et la mise en place de compétences fonctionnelles spécifiques peuvent ainsi être perçues dans ces entreprises comme une source d'avantage concurrentiel (Autio et al., 2000).

L'expérience antérieure du dirigeant dans son secteur d'activité et son expérience personnelle à l'international lui permettent de concrétiser sa vision internationale via la constitution et l'activation de son réseau. Par ailleurs, les connaissances et expériences antérieures réduisent la distance psychique et l'incertitude ; elles jouent un rôle important dans l'augmentation de la vitesse d'apprentissage (Chetty et Campbell-Hunt, 2004). McDougall et al. (1994) soulignent également l'importance de l'expérience de l'entrepreneur dans les affaires internationales, dans la détection d'opportunités internationales et la création d'entreprises qui vont tout de suite à l'international. En raison de leurs compétences uniques (liées à leurs réseaux, à leurs connaissances et leurs parcours), les créateurs des ENI perçoivent et identifient des opportunités que d'autres ne perçoivent pas (Weerawardena et al., 2007). Coeurderoy et al. (2009) mettent en avant l'importance du capital humain acquis par les entrepreneurs sur la base de leur expérience internationale passée pour expliquer la pénétration rapide des marchés étrangers d'entreprises de haute-technologie allemandes et anglaises. Pour Bloodgood et al. (1996), l'expérience passée de travail à l'international dans l'équipe dirigeante d'une entreprise est un antécédent significatif de l'internationalisation des nouvelles entreprises. McDougall et al. (2003) ont montré que l'expérience de travail à l'international et l'expérience de l'équipe à l'origine de la création d'entreprise dans le secteur d'activité de cette dernière, augmentent l'apparition des entreprises nouvelles et internationales. En effet, l'expérience du dirigeant, qui peut être perçue comme la somme des connaissances accumulées au fil des ans, représente le socle sur lequel il va constituer les fondations et les orientations de sa nouvelle entreprise, en particulier à l'international.

Les diverses expériences des entrepreneurs représentent un ensemble de ressources (connaissances des affaires et réseaux personnels) qui peut être utilisé pour la croissance des nouvelles entreprises, en particulier sur les marchés étrangers. D'autres études (Jones et Coviello, 2005 ; Kundu et Katz, 2003) ont montré que les caractéristiques personnelles et cognitives des entrepreneurs ont un impact sur la capacité des nouvelles entreprises à s'internationaliser. 
Les connaissances managériales (liées à l'expérience, au marché, techniques, financières...) jouent aussi un rôle clé pour expliquer l'internationalisation des entreprises (Eriksson et al., 2000 ; Manalova et al., 2002). Les compétences dans le domaine marketing et dans la commercialisation des produits permettent à l'entrepreneur de saisir et d'exploiter des opportunités à l'international.

En synthèse, l'analyse précédente de la littérature nous a permis de mettre en évidence une série de types d'expérience de l'entrepreneur et/ou de son équipe susceptibles d'expliquer l'internationalisation précoce d'une entreprise. Cette expérience peut comporter plusieurs facettes : (i) l'expérience internationale de l'entrepreneur au sens large ; (ii) l'expérience de direction dans le secteur de l'entreprise ; et (iii) les compétences marketing de l'entrepreneur résultant de son expérience professionnelle afin de commercialiser des biens ou des services.

Par ailleurs, le fait d'orienter très fortement son entreprise à l'international permet à l'entrepreneur de ne pas dépendre que de son marché domestique et ainsi d'éviter le phénomène de 'path-dependency' par rapport au marché domestique (Autio et al., 2000). L'entreprise est dès lors plus à même de saisir l'ensemble des opportunités présentes sur les différents marchés à l'étranger ou de tirer pleinement avantage d'une opportunité au niveau international. Pour toutes ces raisons, nous formulons la proposition suivante à tester dans le cadre de cette étude exploratoire.

Proposition : toutes choses étant égales par ailleurs, il existe une relation positive entre l'expérience anté-création de l'entrepreneur et le niveau d'internationalisation de l'entreprise créée ultérieurement par ce dernier.

Après l'examen du rôle de l'expérience de l'entrepreneur dans l'orientation internationale précoce de son entreprise, dans la partie suivante nous présentons la méthodologie adoptée pour apporter une réponse empirique à notre question de recherche.

\section{Méthodologie}

\subsection{Constitution de l'échantillon}

Dans cet article, nous cherchons à vérifier si les différentes expériences accumulées par l'entrepreneur expliquent pourquoi certaines jeunes entreprises orientent majoritairement leurs activités à l'international. L'analyse est centrée sur les activités de vente à l'étranger avec une focalisation sur la région RhôneAlpes, la deuxième région française en termes de commerce extérieur. A cet effet, nous avons élaboré un questionnaire, affiné grâce à une série de dix entretiens approfondis semi-directifs (Evrard et al., 1997) menés auprès de dix 
entrepreneurs de la région Rhône-Alpes ayant créé une entreprise industrielle internationale. Pour mieux appréhender le phénomène étudié, des choix ont été nécessaires, notamment sur le champ d'analyse et le concept 'entreprise nouvelle et internationale'. Suivant Zahra (2005), nous avons choisi d'étudier uniquement les entreprises créées ex-nihilo par des personnes physiques réellement à l'origine de leur création et de leur démarche internationale. Nous avons exclu les entreprises qui ont reçu le soutien direct d'une personne morale dans leur phase d'internationalisation, notamment les entreprises essaimées ou créées à la suite d'une restructuration d'entreprises déjà existantes. En outre, l'étude s'intéresse particulièrement aux entreprises ayant développé une activité de vente à l'international lorsqu'elles étaient âgées d'au plus six ans. Ce seuil est l'une des limites généralement utilisées dans la littérature en entrepreneuriat pour déterminer la jeunesse d'une entreprise (Brush, 1995). Ce seuil a aussi été utilisé dans plusieurs études sur les ENI (Shrader et al., 2000 ; Loane and Bell, 2006). Pour Oviatt et McDougall (1997), si l'internationalisation arrive durant cette période, elle est probablement survenue durant la période de formation de l'entreprise.

Pour constituer notre échantillon, nous avons utilisé la base de données Telexport qui recense les entreprises exportatrices françaises. Nous nous sommes concentrés sur les principaux secteurs de l'industrie française, représentant au total 25 grandes catégories de codes NAF. Nous avons limité l'analyse aux PME au sens européen du terme, soit des entreprises de moins de 250 salariés. Cette limitation a le mérite d'éviter d'inclure dans la population tous les établissements de grande taille ou les filiales de groupes, tout en conservant les jeunes entreprises nouvellement créées. Cette démarche a permis d'identifier une population de 2774 entreprises répondant aux caractéristiques retenues. Dans la mesure où la base de données utilisée ne renseigne pas la date de création, ni son éventuelle appartenance à un groupe, des recherches complémentaires ont été nécessaires. Après vérification auprès de diverses sources (Chambres de commerce et de l'industrie, greffes du tribunal de commerce, contacts directs avec les entreprises), nous avons identifié 488 entreprises auxquelles nous avons adressé un questionnaire. Elles ont été créées entre 1993 et 2000 ; elles ont exercé une activité à l'international entre 1993 et 2002 inclus et appartiennent aux codes NAF sélectionnés. En raison des restrictions occasionnées par la constitution de la base de données, notre démarche s'assimile à une méthode de sélection par choix raisonné (Royer et Zarlowski, 1999), approche très courante dans les recherches en management. Elle repose essentiellement sur le jugement du chercheur. La constitution de l'échantillon se fonde donc sur différents critères propres à la population étudiée et permet de choisir de manière précise les éléments de l'échantillon afin de respecter plus facilement les critères fixés par le chercheur. Au total, 111 questionnaires ont été reçus, soit un taux de retour, après épuration de la base de données (élimination des erreurs de la base de données et des retours à l'envoyeur), de l'ordre de $25 \%$. Dans la population 
de départ à la base de notre échantillon, nous n'avions aucune indication sur la proportion d'entreprises appartenant effectivement à notre objet d'étude. Au total, 68 entreprises sont issues d'une création ex-nihilo après 1993 par un entrepreneur (personne physique) et ont développé une activité à l'international au plus six ans après leur création. Elles représentent $61,2 \%$ du total des questionnaires reçus.

\subsection{Opérationnalisation des variables}

Dans cette section, nous présentons les différentes variables utilisées dans l'étude empirique ainsi que la manière dont elles ont été mesurées.

Internationalisation : comme beaucoup d'études en entrepreneuriat international (McDougall et al., 2003 ; Loane et Bell, 2006), nous avons mesuré l'internationalisation par le pourcentage du chiffre d'affaires réalisé à l'étranger.

Expériences anté-création : les différentes formes d'expérience ont été mesurées via des questions demandant aux répondants d'évaluer les différentes formes d'expériences ou de compétences en rapport avec les activités internationales sur une échelle de Likert de 1 à 5 . Ainsi donc, les différentes variables retenues sont :

- l'expérience dans les affaires internationales (EPAFFIAL),

- l'expérience de direction dans le secteur (EPDIRSEC),

- les connaissances techniques liées au secteur (CNTECSEC),

- les compétences en marketing (CPTCMKTG),

- la formation comportant une dimension internationale (FORMIALE).

Niveau d'internationalisation : Knight and Cavusgil (2004, p. 649) opérationnalisent les entreprises dites 'born-globals' comme les entreprises de moins de 20 ans qui se sont internationalisées dans les trois années après la création et qui ont réalisé au moins $25 \%$ du total des ventes à l'étranger. Cette limite de $25 \%$ a été utilisée dans d'autres études en entrepreneuriat international (Madsen et al., 2000). Bien que cette limite de $25 \%$ soit très largement utilisée et présente l'avantage de faciliter les comparaisons, Knight et Cavusgil (2004) reconnaissent qu'elle est quelque peu arbitraire et qu'elle a été établie en fonction des visées exploratoires de leur recherche. Afin de distinguer parmi les entreprises nouvelles et internationales celles qui dépendent véritablement pour leur activité des marchés étrangers, nous choisissons de porter ce seuil à $50 \%$. Ainsi, nous distinguons mieux les entreprises qui dépendent du marché domestique (phénomène de dépendance par rapport au chemin suivi ou 'pathdependency') de celles pour lesquelles ce n'est pas le cas, mais qui dépendent des marchés étrangers pour leur développement.

Ainsi, pour mesurer l'impact de l'expérience passée sur le niveau de d'internationalisation des jeunes entreprises, nous avons scindé l'échantillon en 
deux groupes distincts en fonction du niveau d'internationalisation. Le premier groupe (Groupe 1, faiblement internationalisé) représente les entreprises dont l'activité de ventes dépend majoritairement du marché français (strictement inférieure à $50 \%$ des ventes à l'international). Le deuxième groupe (Groupe 2 , fortement internationalisé) est composé d'entreprises dont l'activité de ventes dépend majoritairement de l'international (au moins $50 \%$ des ventes à l'étranger).

Variables de contrôle : dans la mesure où l'âge peut expliquer un niveau d'internationalisation plus élevé, nous avons utilisé l'âge de l'entreprise en 2002 (AGEEN2002) comme variable de contrôle. En effet, plus une entreprise est âgée, plus elle est susceptible d'avoir une présence importante à l'étranger.

\subsection{Analyse des données}

Analyses descriptives : partant de ces variables, nous menons d'abord une série d'analyses descriptives, sous la forme de tris à plat. Ces derniers visent à dresser le profil, d'une part, des entrepreneurs en termes d'âge, de sexe, de niveau d'études et de dernière fonction exercée et, d'autre part, des entreprises de l'échantillon en fonction de leurs caractéristiques (secteur d'activité, chiffre d'affaires, personnel, niveau d'internationalisation...).

Analyses explicatives : nous menons ensuite des régressions logistiques visant à déterminer si l'expérience passée de l'entrepreneur explique l'appartenance à l'un des deux groupes d'entreprises : celui des entreprises à dépendance internationale modérée (Groupe 1) ou plutôt celui des entreprises à forte dépendance internationale (Groupe 2). A cet effet, nous effectuons une régression logistique de la variable " niveau d'internationalisation » sur les variables liées à l'expérience susmentionnées, en contrôlant l'âge de l'entreprise.

La méthode de régression « stepwise backward deletion » est utilisée. Toutes les variables liées à l'expérience et aux compétences anté-création de l'entrepreneur, ainsi que la variable de contrôle sont introduites dans le modèle. Les variables explicatives sont successivement éliminées du modèle en fonction de la faible variance partagée avec les prédicteurs. Enfin, le modèle le plus parcimonieux incluant la variable de contrôle est retenu. Toutefois, une analyse de robustesse du modèle est effectuée par comparaison des modèles en se servant de la capacité prédictive du modèle à classifier les observations. 


\section{Résultats}

Dans cette section, nous présentons quelques données relatives aux secteurs et aux parcours des entrepreneurs avant d'aborder les résultats des régressions.

\subsection{Les premières étapes à l'international : entrepreneur et démarrage}

\subsubsection{La répartition des secteurs}

Le Tableau 1 présente la répartition des deux types d'entreprises étudiés en fonction des grandes catégories de codes NAF. Au total, près des deux tiers de l'échantillon (65\% de l'effectif total) appartiennent au groupe 1 (faiblement internationalisé) et dépendent donc majoritairement des activités domestiques et le solde (35\% de l'effectif total) appartient au groupe 2 d'entreprises fortement internationalisées qui dépendent majoritairement des marchés étrangers pour leur activité.

\begin{tabular}{|l|l|l|}
\hline Code NAF & $\begin{array}{l}\text { Groupe 1 'Entreprises } \\
\text { à dépendance } \\
\text { internationale modérée' } \\
-<\text { égal à 50\%; N=44 }\end{array}$ & $\begin{array}{l}\text { Groupe 2 'Entreprises } \\
\text { à forte dépendance } \\
\text { internationale' - } \geq 50 \% \\
\mathrm{~N}=24\end{array}$ \\
\hline $\begin{array}{l}\text { Code } 1: \text { industries alimentaires, tabac, } \\
\text { textiles et de l'habillement }\end{array}$ & $7 \%$ & $7 \%$ \\
\hline $\begin{array}{l}\text { Code } 2: \text { industries chimiques, industries } \\
\text { du caoutchouc et des plastiques, } \\
\text { fabrication d'autres produits minéraux } \\
\text { non métalliques, travail des métaux, } \\
\text { fabrication de machines et équipements }\end{array}$ & $22 \%$ & $26 \%$ \\
\hline $\begin{array}{l}\text { Code 3 : fabrication de machines de } \\
\text { bureau et de matériel informatique, } \\
\text { fabrication de machines et appareils } \\
\text { électriques, fabrication d'équipements } \\
\text { de radio, télévision et communication, } \\
\text { fabrication d'instruments médicaux, de } \\
\text { précision, d'optique, industrie automobile, } \\
\text { fabrication de meubles, industries } \\
\text { diverses }\end{array}$ & $8,5 \%$ & $8,5 \%$ \\
\hline \begin{tabular}{l} 
Code 7 : activités informatiques \\
\hline
\end{tabular} & $9,5 \%$ & $10,5 \%$ \\
\hline
\end{tabular}

Tableau 1 : Répartition des entreprises en fonction des groupes et des secteurs d'activité

Concernant le secteur d'activité, le Tableau 1 indique que 35\% des entreprises de l'échantillon appartiennent au groupe 2 des entreprises dépendant majoritairement de l'international. Cependant, dans les deux groupes on recense à peu près la même proportion d'entreprises quel que soit le secteur d'activité, 
même si le secteur d'activité le plus représenté dans l'échantillon est celui des industries chimiques. Ce résultat suggère que les ENI ne sont pas toujours dans les secteurs high-tech.

Dans cette section, nous présentons quelques données relatives aux parcours des entrepreneurs avant d'aborder les résultats des régressions.

\subsubsection{Le profil des entrepreneurs}

Les entrepreneurs de notre échantillon ont en moyenne 46 ans, cette moyenne variant peu entre les deux groupes (46,4 ans pour ceux du groupe 1 et 45,7 pour ceux du groupe 2). S'ils sont quasiment tous de sexe masculin et de nationalité française (97\% pour ces deux dimensions), $57 \%$ d'entre eux ont un niveau Master. La comparaison du niveau de formation au sein des deux groupes (voir le Tableau 2a) indique que les entrepreneurs du groupe 2 (forte internationalisation) sont approximativement deux fois plus nombreux à disposer d'un niveau Master ( $39 \%$ pour le groupe 1 contre $83 \%$ pour le groupe 2 ) et à provenir d'une école de commerce ou d'ingénieur.

Tableau 2a : comparaison du niveau de formation des entrepreneurs des deux groupes

\begin{tabular}{|l|l|l|}
\hline Niveau d'étude des entrepreneurs (\% par groupe) & $\begin{array}{l}\text { Groupe 1 } \\
\% \text { export : <50\%) }\end{array}$ & $\begin{array}{l}\text { Groupe 2 } \\
\% \text { export } \geq 50 \%\end{array}$ \\
\hline En-dessous baccalauréat & $12 \%$ & $4 \%$ \\
\hline En-dessous du niveau Master & $61 \%$ & $17 \%$ \\
\hline Niveau Master au minium & $39 \%$ & $83 \%$ \\
\hline Diplôme d'école d'ingénieur & $23 \%$ & $46 \%$ \\
\hline Diplôme d'école de commerce & $11 \%$ & $25 \%$ \\
\hline Diplôme de doctorat & $5 \%$ & $12 \%$ \\
\hline
\end{tabular}

Tableau 2b : comparaison des dernières fonctions occupées par les entrepreneurs des deux groupes

\begin{tabular}{|l|l|l|}
\hline Dernière fonction occupée (en $\%)$ & $\begin{array}{l}\text { Groupe } 1 \\
\% \text { export : <50\%) }\end{array}$ & $\begin{array}{l}\text { Groupe } 2 \\
\% \text { export } \geq 50 \%\end{array}$ \\
\hline PDG, DG, Directeur de division, General Manager & $13 \%$ & $34 \%$ \\
\hline $\begin{array}{l}\text { Poste de direction ou de responsable technique, de } \\
\text { production ou de R \& D }\end{array}$ & $21 \%$ & $8 \%$ \\
\hline Poste de direction commerciale & $13 \%$ & $16 \%$ \\
\hline Chef de projet, chef de mission, cadre, responsable & $18 \%$ & $30 \%$ \\
\hline Technicien ou commercial & $35 \%$ & $4 \%$ \\
\hline Fonctionnaire & 0 & $8 \%$ \\
\hline Total & $100 \%$ & $100 \%$ \\
\hline \multicolumn{2}{|l|}{ Tableau 2c : comparaison de la correspondance entre la formation suivie et le } \\
\hline
\end{tabular}




\begin{tabular}{|l|l|l|}
\hline $\begin{array}{l}\text { Formation dans le même domaine que l'entreprise créée } \\
\text { (en } \%)\end{array}$ & $\begin{array}{l}\text { Groupe 1 } \\
\% \text { export : <50\%) }\end{array}$ & $\begin{array}{l}\text { Groupe 2 } \\
\% \text { export } \geq 50 \%\end{array}$ \\
\hline $\begin{array}{l}\text { Formation technique sans lien avec le domaine d'activité } \\
\text { de l'entreprise }\end{array}$ & $12 \%$ & $0 \%$ \\
\hline Autodidacte & $8 \%$ & $0 \%$ \\
\hline $\begin{array}{l}\text { Entrepreneurs ayant suivi uniquement une formation en } \\
\text { gestion }\end{array}$ & $23 \%$ & $22 \%$ \\
\hline $\begin{array}{l}\text { Formation spécialisée dans le domaine d'activité de } \\
\text { l'entreprise }\end{array}$ & $25 \%$ & $70 \%$ \\
\hline $\begin{array}{l}\text { Formation technique généraliste liée au domaine } \\
\text { d'activité de l'entreprise }\end{array}$ & $32 \%$ & $8 \%$ \\
\hline Total & $100 \%$ & $100 \%$ \\
\hline
\end{tabular}

Tableau 2 : Comparaison entre les deux groupes

L'analyse du Tableau $2 \mathrm{~b}$ rend compte de l'expérience anté-création des entrepreneurs en comparant les dernières fonctions occupées par ces derniers. Si au niveau de l'échantillon global, $77 \%$ des entrepreneurs ont occupé des fonctions de manager ou de cadre avant de développer une activité entrepreneuriale, il existe cependant des différences notables en termes de dernière fonction occupée entre les deux groupes. Plus d'un tiers (35\%) des entrepreneurs du groupe 1 ont occupé comme dernière fonction des postes sans responsabilité contre $4 \%$, soit un seul entrepreneur du groupe 2. Un tiers environ des entrepreneurs du groupe 2 (le plus internationalisé) a occupé une fonction de direction et la même proportion a exercé une fonction de chef de projet ou de responsable à un niveau cadre.

Toutes proportions gardées, les entrepreneurs du groupe 2 (le plus internationalisé) sont 3,4 fois plus nombreux à avoir occupé une fonction de direction et 1,7 fois plus à avoir occupé une fonction de chef de projet ou de responsable à un niveau cadre par rapport à ceux du groupe 1. Par contre, un cinquième des entrepreneurs du groupe 1 (le moins internationalisé) ont occupé des fonctions à responsabilité purement technique ; ils sont 2,7 fois plus nombreux que les entrepreneurs du groupe 2. Environ un sixième seulement des entrepreneurs des deux groupes ont occupé des postes à responsabilité purement commerciale. II ressort de la comparaison que les entrepreneurs des deux groupes ont des expériences professionnelles anté-création très différentes.

Le Tableau 2c, qui examine la cohérence entre le type d'entreprises créées et la nature de la formation des entrepreneurs, présente une situation très contrastée entre les deux groupes. Près d'un quart des entrepreneurs (23\% et $22 \%$ ) ont uniquement suivi une formation en gestion, dont la totalité au niveau Master en école de commerce pour les entrepreneurs du groupe 2. Près de trois quarts des entrepreneurs du groupe $2(70 \%)$ ont une formation spécialisée dans le 
même domaine d'activité que l'entreprise créée contre un quart seulement pour les entrepreneurs du groupe 1 . Un tiers des entrepreneurs du groupe 1 ont une formation technique ayant un lien avec les activités de l'entreprise créée (qui reste une formation généraliste) et $12 \%$ d'entre eux ont une formation technique n'ayant rien à voir avec le domaine d'activité de l'entreprise créée.

\subsubsection{Démarrage international de l'entreprise : le profil des entreprises}

II ressort de l'enquête que les entreprises étudiées sont en moyenne âgées de six ans, avec une expérience moyenne à l'international de cinq ans. En outre, si le temps moyen entre la création de l'entreprise et la réalisation de la première vente à l'international est de 14 mois, près de huit entreprises sur dix (78\%) s'étaient internationalisées durant l'année de leur création. Un examen plus approfondi montre également que les entreprises du groupe 2 (à forte dépendance envers l'international) sont parties majoritairement à l'international dès l'année de leur création $(70,7 \%)$, tandis que celles du groupe 1 (forte internationalisation) y sont allées un an ou plus après la création de l'entreprise. Ainsi, dans notre échantillon, les entreprises les plus internationalisées sont également celles qui ont démarré le plus tôt le processus d'internationalisation (dès la création de l'entreprise).

\subsection{Le lien entre l'expérience passée de l'entrepreneur et le niveau d'internationalisation}

Le Tableau 3 présente les deux modèles de régression testés : le modèle de 1 à 4 prédicteurs et le modèle de 1 à 6 prédicteurs comportant deux variables supplémentaires : la formation à l'international (FORMIALE) et la connaissance technique liée au secteur d'activité de l'entreprise créée (CNTECSEC). Ce tableau indique que les signes des coefficients sont inchangés dans les deux modèles, de même que le niveau de significativité. Des deux facteurs ajoutés dans le modèle 2 , seule la « formation comportant une dimension internationale (FORMIALE) est significative avec un signe positif. Ce résultat suggère que les entrepreneurs ayant suivi une formation comportant une dimension internationale créent des entreprises fortement internationalisées (dépendant fortement des marchés étrangers). 


\begin{tabular}{|c|c|c|c|c|c|c|}
\hline & Modèle 1 & $\begin{array}{l}\text { Signe } \\
\text { attendu }\end{array}$ & $\begin{array}{l}\text { Signe } \\
\text { obtenu }\end{array}$ & Modèle 1 & Modèle 2 & Modèle 2 \\
\hline Variable & B & & & $\operatorname{Exp}(B)$ & B & $\operatorname{Exp}(B)$ \\
\hline Epaffial & $\begin{array}{l}1.363^{* * *} \\
(.001)\end{array}$ & + & + & 3.907 & $\begin{array}{l}1.100 * * * \\
(0.008)\end{array}$ & 3.005 \\
\hline Epdirsec & $\begin{array}{l}-.569 \text { ** } \\
(.046)\end{array}$ & + & - & .566 & $\begin{array}{l}-.667^{* *} \\
(.037)\end{array}$ & .513 \\
\hline Cntecsec & & & & & $\begin{array}{l}.526 \\
(.123)\end{array}$ & 1.692 \\
\hline Cptcmktg & $\begin{array}{l}-.699^{* *} \\
(.035)\end{array}$ & + & - & .497 & $\begin{array}{l}-.730 * * \\
(.037) \\
\end{array}$ & .482 \\
\hline Formiale & & & & & $\begin{array}{l}.485^{*} \\
(.085)\end{array}$ & 1.625 \\
\hline Ageen2002 & $\begin{array}{l}-.337^{* *} \\
(.013)\end{array}$ & & & 3.234 & $\begin{array}{l}-.374^{* *} \\
(.012)\end{array}$ & .688 \\
\hline Constante & $\begin{array}{l}1.174 \\
(.349)\end{array}$ & & & & $\begin{array}{l}-.871 \\
(.616)\end{array}$ & .418 \\
\hline -2log-likelihood ratio & 62.846 & & & & 57.200 & \\
\hline $\begin{array}{l}\text { Cox et Snell R } \\
\text { square }\end{array}$ & .312 & & & & .367 & \\
\hline Nagelkerke R square & .429 & & & & .505 & \\
\hline $\begin{array}{l}\text { Hosmer-Lemeshow } \\
\text { test ( } p \text { value) }\end{array}$ & .981 & & & & .904 & \\
\hline
\end{tabular}

Tableau 3: Régression logistique - modèles 1 et 2

Le Tableau 4 qui compare les deux modèles en termes de pouvoir de classification des données, indique que l'ajout de 2 prédicteurs supplémentaires au modèle 1 n'entraîne qu'une augmentation marginale d'environ $2 \%$ du pouvoir prédictif du modèle. C'est pourquoi, la présentation et la discussion des résultats se limiteront au modèle 1 , le plus parcimonieux.

\begin{tabular}{|c|c|c|c|c|c|c|c|c|c|}
\hline \multicolumn{5}{|c|}{$\begin{array}{l}\text { Classification proposée par le modèle à } 4 \\
\text { prédicteurs }\end{array}$} & \multicolumn{5}{|c|}{$\begin{array}{l}\text { Classification proposée par le modèle à } 6 \\
\text { prédicteurs }\end{array}$} \\
\hline \multicolumn{2}{|l|}{ Observé } & \multicolumn{3}{|c|}{ Attendu } & \multicolumn{2}{|l|}{ Observé } & \multicolumn{2}{|c|}{ Attendu } & \multirow[b]{2}{*}{$\begin{array}{l}\text { Pourcentage } \\
\text { classification } \\
\text { correcte }\end{array}$} \\
\hline & & \multicolumn{2}{|c|}{$\begin{array}{l}1 \text { si \% chiffre } \\
\text { d'affaires à } \\
\text { l'international } \\
<50 \text { sinon } 2\end{array}$} & $\begin{array}{l}\text { Pourcentage } \\
\text { classification } \\
\text { correcte }\end{array}$ & & & \multicolumn{2}{|c|}{$\begin{array}{l}1 \text { si } \% \text { chiffre } \\
\text { d'affaires à } \\
\text { l'international } \\
<50 \text { sinon } 2\end{array}$} & \\
\hline & & 1 & 2 & 1 & & & 1 & 2 & 1 \\
\hline \multirow{2}{*}{$\begin{array}{l}1 \text { si \% chiffre } \\
\text { d'affaires à } \\
\text { l'international } \\
<50 \text { sinon } 2\end{array}$} & 1 & 39 & 5 & 88,6 & \multirow{2}{*}{$\begin{array}{l}1 \text { si \% chiffre } \\
\text { d'affaires à } \\
\text { l'international } \\
<50 \text { sinon } 2\end{array}$} & 1 & 40 & 4 & 90,9 \\
\hline & 2 & 8 & 16 & 66,7 & & 2 & 7 & 17 & 70,8 \\
\hline $\begin{array}{l}\text { Pourcentage } \\
\text { global }\end{array}$ & & & & 80,9 & $\begin{array}{l}\text { Pourcentage } \\
\text { global }\end{array}$ & & & & 83,8 \\
\hline
\end{tabular}

Tableau 4 : Comparaison des modèles à 4 et 6 prédicteurs 
II ressort du Tableau 3 que le modèle est globalement significatif avec un test de spécification du modèle significatif au seuil de 0,1\% (chi-carré : 25.452). Le pseudo R2 de Nagelkerke est de 0,429 , ce qui est élevé pour une variable dépendante qualitative. Le ratio du maximum de vraisemblance s'élève à 64,875 débouchant sur un chi-carré de 25,452 avec 4 degrés de liberté très significatif au seuil de $1 \%$. Ce résultat suggère fortement le rejet de l'hypothèse nulle stipulant que tous les paramètres du modèle, à l'exception de la constante, valent zéro. De même, le degré de signification du test de Hosmer-Lemeshow $(0,981)$ est supérieur à 0,05 , ce qui indique un bon ajustement du modèle aux données. Globalement, le modèle prédit donc correctement l'appartenance à une catégorie de niveau d'internationalisation à $80,9 \%$ (tableau 4 ).

L'examen des paramètres estimés du modèle indique qu'en utilisant l'âge de l'entreprise (AGEEN2002) comme variable de contrôle, au seuil de significativité de $1 \%$, la probabilité qu'une jeune entreprise appartienne à la catégorie des entreprises réalisant au moins la moitié de leur chiffre d'affaires à l'international croîtavec l'expérience antérieure de l'entrepreneur dans les affaires internationales (EPAFFIAL). En revanche, au seuil statistique de $5 \%$ cette probabilité décroît tant avec l'expérience de direction dans le secteur (EPDIRSEC) que les compétences en marketing (CPTMKTG).

\section{Discussion et conclusion}

Dans cet article, nous avons postulé une relation positive entre l'expérience anté-création de l'entrepreneur et le niveau d'internationalisation de l'ENI créée. A cet effet, dans le modèle retenu le plus parcimonieux nous avons examiné l'incidence des différentes déclinaisons de cette expérience : (i) l'expérience dans les affaires internationales ; (ii) l'expérience de direction dans le secteur et (iii) les compétences en marketing. Les résultats de la régression logistique indiquent que seule l'expérience dans les affaires internationales présente une relation positive avec le niveau élevé d'internationalisation de l'entreprise créée, les deux autres variables présentant une relation négative. Nous proposons ci-après une interprétation possible de ces résultats.

Nous estimons que l'expérience du secteur se retrouve d'abord au niveau de la formation, puis orientée vers des fonctions de management généraliste de haut rang associées généralement à un niveau d'études plus élevé (carrière plus avancée). Par ailleurs, les affaires internationales reflètent davantage l'expérience de direction des activités de l'entreprise, y compris à l'international. Par contre, dans le groupe 1, il y a une plus forte expérience de direction du secteur, profil plus technique uniquement où il y a une plus forte proportion de direction technique. Ainsi donc, " l'expérience dans les affaires internationales » permet de différencier le groupe 'fortement international' du groupe 'faiblement international'. 'L'expérience dans les affaires internationale' principalement, prédit 
si l'entreprise sera fortement internationalisée ou pas, c'est-à-dire si l'entreprise dépend majoritairement de ses activités internationales. Ce résultat suggère un lien direct entre le niveau d'expérience dans les affaires internationales et le niveau d'internationalisation de l'entreprise (appartenance au groupe des entreprises fortement internationalisées). Ce groupe se différencie par une 'expérience dans les affaires internationales' plus importante que le premier. D'une manière générale, les entrepreneurs dont l'entreprise dépend majoritairement de l'international pour son activité ont un niveau de formation supérieure, et pour une majorité d'entre deux dans le même secteur d'activité que l'entreprise créée. Ceci leur permet d'accéder plus facilement à des postes de management à responsabilité, que ce soit au niveau d'une direction générale ou pour le développement de projets. Ce niveau de responsabilité leur permet d'être en contact avec les marchés étrangers, d'acquérir une expérience solide dans les affaires internationales et ainsi de développer très fortement leur activité à l'international dès l'année de création de l'entreprise.

Contrairement à nos attentes, les coefficients des variables 'expérience de direction dans le secteur d'activité de l'entreprise créée' et 'compétences marketing' sont négatifs. Ces résultats invalident notre présupposé indiquant que les variables 'expérience de direction dans le secteur d'activité de l'entreprise créée' et 'compétences marketing' expliquent positivement le niveau d'internationalisation. Un fort niveau d'expérience dans ces deux domaines permet de prédire l'appartenance au groupe 1, c'est-à-dire les entreprises dépendant majoritairement de leur marché domestique. Les entrepreneurs qui se sont écartés de leur marché domestique et qui ont suivi de façon prépondérante la voie internationale sont moins expérimentés dans ces deux domaines de façon significative. Ce résultat pourrait s'expliquer si l'on analyse de manière approfondie l'expérience et la formation des entrepreneurs.

En effet, les entrepreneurs du groupe 1 (entreprises dépendant plus du marché domestique) ont occupé des fonctions de direction, mais plus dans des fonctions liées à des aspects techniques et à un secteur d'activité qui correspond au domaine d'activité de l'entreprise créée. Ils disposent de moins d'expérience de direction générale ou de management par rapport à des projets ou à des missions. Globalement, ils ont un niveau de formation moins élevé qui ne leur a pas permis d'acquérir une expérience internationale et d'arriver dans leur parcours professionnel à de hautes fonctions de direction. De par leur expérience à un niveau de commercial pur ou de technicien, ils ont pu acquérir des compétences liées au marketing, mais un niveau qui reste très opérationnel. Par contre, les entrepreneurs dotés d'une véritable expérience de management (au niveau d'une direction ou de projets) n'ont pas développé des compétences liées aux techniques de marketing. Les entrepreneurs du groupe 1 n'ont pas été confrontés aux marchés étrangers ni directement aux problématiques internationales. Ils n'ont pas l'expérience de terrain d'un responsable export ou d'un chef de zone. Or 
l'international exige un apprentissage récursif et progressif (Johanson et Vahlne, 1977, 1990) qui n'existe ni au niveau organisationnel, ni au niveau individuel dans les entreprises du groupe 1.

Nos résultats suggèrent tout d'abord que toutes les ENI n'ont pas un comportement homogène à l'international. L'expérience passée de l'entrepreneur (dans les affaires internationales, de direction dans le secteur et au niveau marketing) permet d'expliquer et de distinguer le niveau d'internationalisation des entreprises dont l'activité dépend majoritairement des marchés étrangers. Ce résultat est à rapprocher, sur le plan théorique, des analyses de McDougall et al. (1994) et Madsen et Servais (1997) et sur le plan empirique de McDougall et al. (2003) car il met en avant l'expérience passée du secteur et, en particulier, celle dans les affaires internationales pour expliquer le développement international des jeunes entreprises. Même si toutes les entreprises de notre échantillon sont internationalisées six ans ou moins après la création, certaines sont beaucoup plus internationalisées que d'autres. Un élément explicatif de cette différence tient à l'expérience acquise précédemment à la création dans les affaires internationales. Ces résultats sont à rapprocher de ceux de Coeurderoy et al. (2009) soulignant le rôle du capital humain acquis par les entrepreneurs sur la base de leur l'expérience internationale passée pour expliquer la pénétration rapide des marchés.

Par ailleurs, des entrepreneurs même expérimentés (dans le management ou le marketing) n'ont pas forcément les compétences nécessaires pour orienter fortement leur entreprise à l'international et ne plus être pris totalement par le phénomène de 'path-dependency' du marché domestique.

D'un point de vue managérial, nos résultats montrent l'importance d'évaluer - chez les jeunes entreprises qui souhaitent s'internationaliser rapidement - l'expérience et les compétences nécessaires pour se développer de manière significative sur les marchés étrangers. L'influence de l'expérience de l'équipe entrepreneuriale est à prendre en considération, notamment pour évaluer le potentiel de croissance internationale des entreprises. Le diagnostic export d'une jeune entreprise internationale doit analyser les différents types d'expérience acquis par les entrepreneurs ; ceci permet d'analyser le type de capital humain (Coeurderoy et al., 2009) sur lequel l'entreprise peut s'appuyer pour s'internationaliser.

Cependant, notre travail est à évaluer en tenant compte de ses limites. Tout d'abord, nous nous basons essentiellement sur le jugement des entrepreneurs et nous ne nous focalisons que surl'entrepreneur et non sur l'équipe entrepreneuriale. Ensuite, en adoptant une approche multi-activité portant sur le niveau régional, nous ne pouvons pas en généraliser les résultats au niveau national, cette limite étant accentuée par la faible taille de l'échantillon étudié. Par ailleurs, nous évaluons le niveau d'internationalisation seulement par le niveau des ventes à 
l'international, alors que d'autres critères tels les zones géographiques couvertes ou les modes d'entrée, pourraient également être pris en compte.

Nos résultats viennent conforter le développement théorique relatif à l'expérience de l'entrepreneur d'Oviatt et McDougall (1994) dans leur article fondateur du champ de l'entrepreneuriat international. Toutes ces limites sont autant de pistes pouvant alimenter des recherches futures.

\section{Bibliographie}

Autio E., Sapienza K.-J. \& Almeida J.-G. (2000), « Effects of age at entry, knowledge intensity, and imitability on international growth », Academy of Management Journal, Vol. $43, N^{\circ} 5$, p. 909-924.

Autio E. (2005), « Creative tension : the significance of Ben Oviatt's and Patricia McDougall's article "toward a theory of international new ventures ", Journal of International Business Studies, Vol. 36, № 1, p. 9-19.

Brush B. (1995), "International entrepreneurship : the effect of firm age on motives for internationalization", Garland Studies in Entrepreneurship, University of Maine.

Cabrol M., Favre-Bonté V.-A. \& Fayolle (2009), « The influence of the entrepreneur's network on the internationalization of French young firms ", International Journal of Entrepreneurship and Innovation, Vol. 10, № 3 , p. 213-221.

Chetty S. et Campbell-Hunt C. (2004), «A strategic approach to internationalization : a traditional versus a 'born-global' approach », Journal of International Marketing, Vol. 12, $\mathrm{N}^{\circ}$ 1, p. $57-81$.

Eriksson K., Majkgard A. et Deo Sharma D. (2000), « Path dependence and knowledge development in the internationalization process », Management International Review, Vol. $40, \mathrm{~N}^{\circ} 4$, p. 307-328.

Johanson J. et Vahlne J.-E. (1990), « The mechanism of internationalization », International Marketing Review, Vol. 7, $\mathrm{N}^{\circ}$ 4, p. 11-24.

Jones M.-V. et Coviello N.-E. (2005), " Internationalisation : conceptualising an entrepreneurial process of behaviour in time », Journal of International Business Studies, Vol. 36, p. 284-303.

Knight G. et Cavusgil T.-S. (2004), « Innovation, organizational capabilities, and the bornglobal firm », Journal of International Business Studies, Vol. 35, p. 124-141.

Kundu S.-K. et Katz J. (2003), « Born-international SMEs: bi-level impacts of resources and intentions », Small Business Economics, Vol. 20, 25-47.

Loane S. et Bell J. (2006), « Rapid internationalisation among entrepreneurial firms in Australia, Canada, Ireland and New Zealand: An extension to the network approach » International Marketing Review, Vol. 23, № 5, p. 467-485.

Madsen T.-K. et Servais P. (1997), «The internationalisation of born globals : an evolutionary process ? », International Business Review, Vol. 6, № 6, p. 561-583.

Madsen T.-K., Rasmussen E. and Servais P. (2000), « Differences and similarities between born globals and other types of exporters », Advances in International Marketing, Vol. 10, p. $247-265$.

Manalova T.-S., Brusch C.-G., Edelmal L.-F. et Greene P.-G. (2002), « Internationalisation of small firms », International Small Business Journal, Vol. 20, $\mathrm{N}^{\circ}$ 1, p. 9-31.

Mcdougall P.-P., Shane S. et Oviatt B.-M. (1994), « Explaining the formation of international new ventures: the limits of theories from international business research », Journal of Business Venturing, Vol. 9, p. 469-87.

McDougall P.-P., Oviatt B.-M. et Shrader R.-C. (2003), « A comparison of international and domestic new ventures ", Journal of International Entrepreneurship, Vol. 1, n 1, p. 
59-82.

McKinsey et Co (1993), « Emerging exporters : Australia's high value-added manufacturing exporters », Melbourne, Australian Manufacturing Council.

Moen O. (2002), "The born globals : a new generation of small European exporters », International Marketing Review, Vol. 19, № 2, p. 156-175.

Oviatt B.-M. et McDougall P.-P. (1994), « Toward a theory of international new ventures », Journal of International Business Studies, Vol. 24, p. 45-64.

Oviatt B.-M. and McDougall P.-P. (1997), « Challenges for internationalisation process theory: The case of international new ventures », Management International Review, Vol. $37, N^{\circ} 2$, p. 85-99.

Oviatt B.-M. et McdDougall P.-P. (2005), « The internationalization of entrepreneurship », Journal of International Business Studies, Vol. 36, № 1, p. 2-8.

Rialp A., Rialp J. et Knight G.-A. (2005), « The phenomenon of early internationalizing firms : what do we know after a decade (1993-2002) of scientific inquiry ? ", International Business Review, Vol. 14, p. 147-166.

Royer I. et Zarlowski P. (1999), « Le design de la recherche », dans Thiétart R.-A. et al., Méthodes de recherche en Management, Dunod, Paris, p. 139-168.

Servais P. et Rasmussen E.-S. (2000), « Different types of international new ventures », Academy of International Business, conférence annuelle, Novembre, Phoenix, p. 1-27. Shrader R.-C., Oviatt B.-M. et Mcdougall P.-P. (2000), « How new ventures exploit tradeoffs among international risk factors : lessons of the accelerated internationalization of the $21^{\text {st }}$ century », Academy of Management Journal, Vol. 43, № 6, p. 1226-1247.

Weerawardena J., Mort G.-S., Liesch P.-W. et Knight G. (2007), « Conceptualizing accelerated internationalization in the born global firm : a dynamic capabilities perspective», Journal of World Business, Vol. 42, p. 294-306.

Zahra S.-A. (2005), " A theory of international new ventures : a decade of research », Journal of International Business Studies, Vol. 36, p. 20-28. 